\title{
Multi-wavelength emission from dark matter annihilation processes in galaxy clusters using cosmological simulations
}

\section{Remudin Reshid Mekuria*}

E-mail: remudin.mekurialstudents.wits.ac.za

\section{Sergio Colafrancesco}

E-mail: sergio.colafrancesco@wits.ac.za

\section{Andreas Faltenbacher}

E-mail: Andreas.Faltenbacherewits.ac.za

\section{Paolo Marchegiani}

E-mail: paolo.marchegiani@wits.ac.za

School of Physics, University of the Witwatersrand, Private Bag 3, WITS-2050, Johannesburg, South Africa

Based on the Marenostrum-MultiDark SImulation of galaxy Clusters (MUSIC-2) we develop semi-analytical models which provide multi-wavelength emission maps generated by dark matter (DM) annihilation processes in galaxy clusters and their sub-halos. We focus on radio and gamma-ray emission maps from neutralino DM annihilation processes testing two different neutralino masses, $\mathrm{M}_{\chi}, 35 \mathrm{GeV}$ and $60 \mathrm{GeV}$ along with two different models of magnetic fields. A comparison of the radio flux densities from our DM annihilation model with the observed diffuse radio emission from the Coma cluster shows that they are of the same order of magnitude. We determine the DM densities with a Smoothed Particle Hydrodynamics (SPH) kernel. This enables us to integrate the DM annihilation signal along any given line-of-sight through the volume of the cluster. In particular it allows us to investigate the contribution of sub-halos to the DM annihilation signal with very high resolution. Zooming in on a subset of high mass-to-light ratio DM sub-halos, i.e. DM sub-halos with very low baryon content, we demonstrate that such targets can generate prominent annihilation signals. The radial distribution of high mass-to-light ratio (M/L) DM sub-halos is more strongly peaked at $\approx \mathrm{R}_{200 \text { crit }}$ compared to the distribution of all sub-halos which may suggest that the search for DM annihilation signals from sub-halos in clusters is most promising at $\mathrm{R}_{200 \text { crit }}$. The radio flux densities from DM sub-halos are well within the sensitivity limit of Square Kilometer Array (SKA) with an integration time of 1000 hours and unlike clusters their gamma-ray spectrum is seen to be dominated by pion decay over a wide range of gamma-ray energies. Our model makes clear predictions for future radio and gamma-ray observations of the DM annihilation signal in clusters and their sub-halos.

4th Annual Conference on High Energy Astrophysics in Southern Africa

25-27 August, 2016

South African Astronomical Observatory (SAAO), Cape Town, South Africa 


\section{Introduction}

Galaxy clusters are the largest gravitationally bound structures in the universe composed of galaxies 3\%, intra-cluster medium (ICM) $12 \%$ and DM 85\% [1]. Numerical simulations show that galaxy clusters form through grouping of smaller objects or "accretion" of clumps along the filaments and rarely through "mergers" with larger clusters which is also known as "bottom-up" scenario. The largest clusters often form at the intersection of these filamentary structures [2] and the formation of these structures is driven by DM [3].

Galaxy clusters are of great importance as they manifest the large scale structure formation of the universe and that of DM. A type of cold DM particles which are weakly interacting massive particles (WIMPs) known as neutralinos are believed to constitute DM [4]. The neutralinos are Majorana type particles hence they are their own anti-particle. When two such particles come close, they are expected to annihilate and produce electromagnetic emission over a broad spectral range. In the following we will study the multi-wavelength emission from DM annihilation in our simulations.

\section{Numerical method}

In order to determine the DM annihilation flux from neutralino $\chi$ we employ the MUSIC-2 simulation [5], which is a high resolution hydrodynamical, zoomed-in simulation of 282 galaxy clusters. The mass resolution of the simulation is $9.01 \times 10^{8} \mathrm{~h}^{-1} \mathrm{M}_{\odot}$ for DM. Figure 1 shows the density profile of one specific cluster. The DM, gas and star density profiles are given in red (solid curve), blue (dot-dashed curve) and green (dotted curve), respectively. At the outskirts of the cluster the slope of the DM and gas density profiles are very similar. The dominance of the stellar component in the inner part is a manifestation of the overcooling problem, a well known feature in current cosmic simulations due to shortcomings in the prescription of feedback mechanism.

The evaluation of the DM annihilation flux from neutralino $\chi$ involves a product of two terms: the term $A_{D M}$, the particle physics part, that depends on the particle physics model of the DM, and the cosmological part which involves a line-of-sight volume integral of the square of the DM density evaluated in each cube as given below

$$
\Phi=A_{D M} \int_{\text {l.o.s }} \rho_{\chi}^{2} d V
$$

where $\rho_{\chi}$ is the DM density.

Taking the DM particle information at redshift zero we determined the density $\rho_{i}$ of the $i^{\text {th }}$ DM particle using Smoothed Particle Hydrodynamics (SPH) kernel, which evaluates the density at each DM particle position taking in to account the 44 neighboring particles [6]. Once the densities are calculated at each particle position the square of the DM densities is obtained by integrating along the line-of-sight. In Fig. 2 we give the projected map of the square of the DM density of a cluster $2 \mathrm{Mpc}$ across that are obtained using SPH kernel. The brightest colors in the map clearly show enhanced DM density substructures which are required to correctly determine emissions from the DM annihilation process in galaxy clusters.

\footnotetext{
* Speaker.
} 


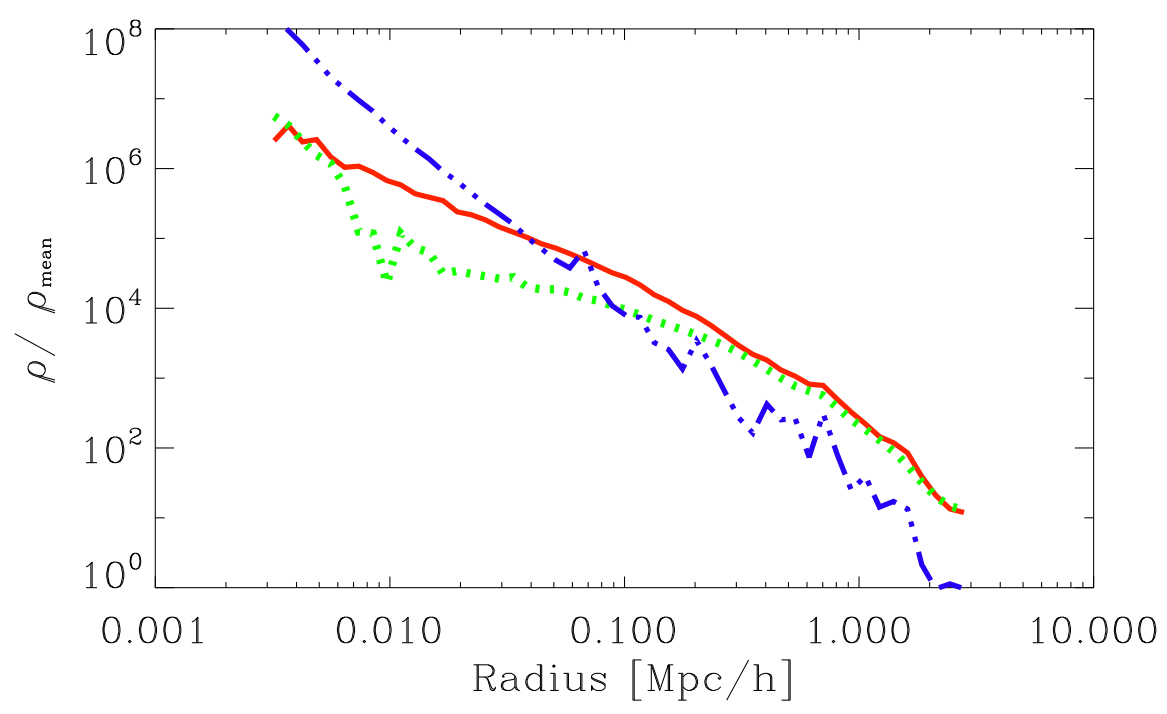

Figure 1: The density profile of DM, gas and stars of a cluster in red (solid curve), blue (dot-dashed curve) and green (dotted curve), respectively.

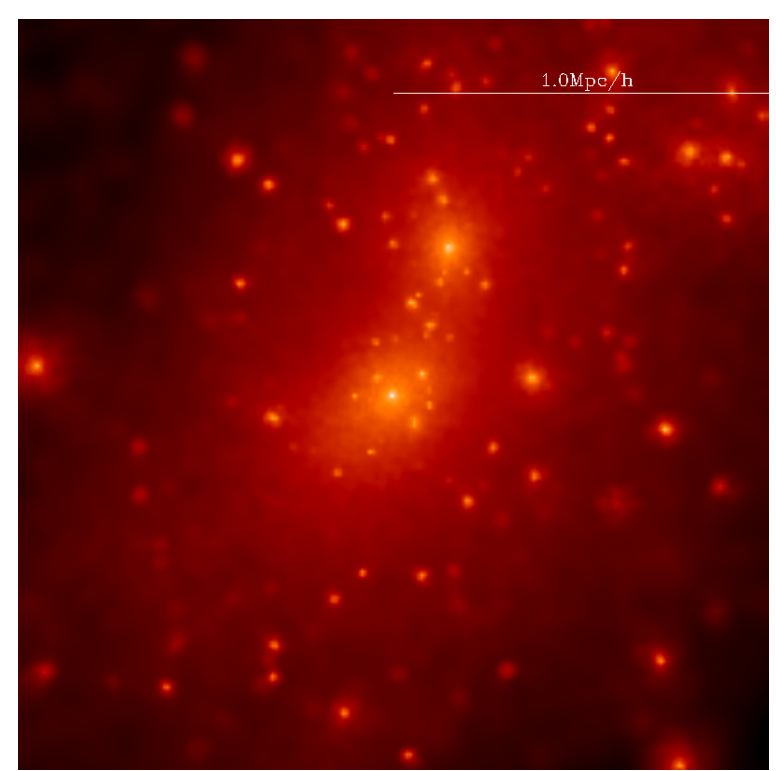

Figure 2: Projected map of the square of the DM density of a cluster $2 \mathrm{Mpc}$ across obtained using SPH kernel.

\subsection{Magnetic field of a galaxy cluster}

We have considered two magnetic field models in order to determine the magnetic field in each cube:

(i) Model A is a model that evaluate the magnetic field strength from the density of DM as in [7]. 
The magnetic field profile is given as

$$
B(r)=B_{0}\left(1+\left(r / r_{s}\right)^{2}\right)^{-q_{b}},
$$

where $B_{0}$ is the central magnetic field strength, $r$ is the radial distance from cluster center, $r_{s}$ is the scale radius of the DM density profile and $q_{b}$ is a scaling exponent chosen to be 0.5 . As we wanted to determine the magnetic field strength in each cube of size of $10 \mathrm{kpc}$, for each point in the simulation box we determine their respective densities which will allow us to calculate the magnetic field as a function of the DM density in each cube.

(ii) Model $\mathrm{B}$ uses the best observational value for a central magnetic field in a coma cluster equal to $4.7 \mu \mathrm{G}$ (at electron number density of $3.44 \times 10^{-3} \mathrm{~cm}^{-3}$ ) from [8]. The magnetic field $B$ of the galaxy cluster is related to the electron number density $n_{e}$ by

$$
B=4.7 \mu G\left(n_{e} / 3.44 \times 10^{-3} \mathrm{~cm}^{-3}\right)^{0.5} .
$$

We evaluated the electron number density $n_{e}$ in $\mathrm{cm}^{-3}$ as in [5],

$$
n_{e}=N_{e} \rho_{\mathrm{gas}}\left(1-Z-Y_{\mathrm{He}}\right) / m_{p},
$$

where $N_{e}, \rho_{\mathrm{gas}}, Z, Y_{\mathrm{He}}$ and $m_{p}$ are the number of ionized electrons per hydrogen atoms, gas density, metallicity, helium concentration and proton mass, respectively.

Figure 3 shows the magnetic field profile for these two models. In the inner region of the cluster the electron number density increases very fast causing the magnetic field as in the solid curve in green color to rise fast. The magnetic model shown in dotted curve in red color shows a very close value to the central magnetic field value of $B_{0}=5.0 \mu \mathrm{G}$ except in the outer region of the cluster where it falls fast. In the following, we will employ these two magnetic field models in calculating the emission expected from the DM annihilation process.

\subsection{Radio and Gamma-ray emission from DM annihilation processes}

\section{Synchrotron radiation}

Diffuse radio emission has been observed in large number of clusters. The distribution of magnetic field, and energetic electrons in the wider region of galaxy cluster is believed to be produced by synchrotron radiation. Synchrotron radiation is emitted when an electron gyrates about a magnetic field lines. For an amplitude of magnetic field $B$ the average power radiated by electrons with an energy $E$ from synchrotron processes at frequency $v$ is formulated as in [9].

$$
P_{\text {synch }}(v, E, r, z)=\int_{0}^{\pi} d \theta \frac{\sin \theta^{2}}{2} 2 \pi \sqrt{3} r_{e} m_{e} c v_{g} F_{\text {synch }}\left(\frac{\kappa}{\sin \theta}\right)
$$

where $m_{e}$ is the electron mass, $v_{g}=\frac{e B}{2 \pi m_{e} c}$ is the non-relativistic gyro-frequency, $r_{e}=\frac{e^{2}}{m_{e} c^{2}}$ is the classical electron radius, and the quantities $\kappa$ and $F_{\text {synch }}$ are defined as

$$
\kappa=\frac{2 v(1+z)}{3 v_{0} \gamma^{2}}\left(1+\left(\frac{\gamma v_{p}}{v(1+z)}\right)^{2}\right)^{\frac{3}{2}}
$$




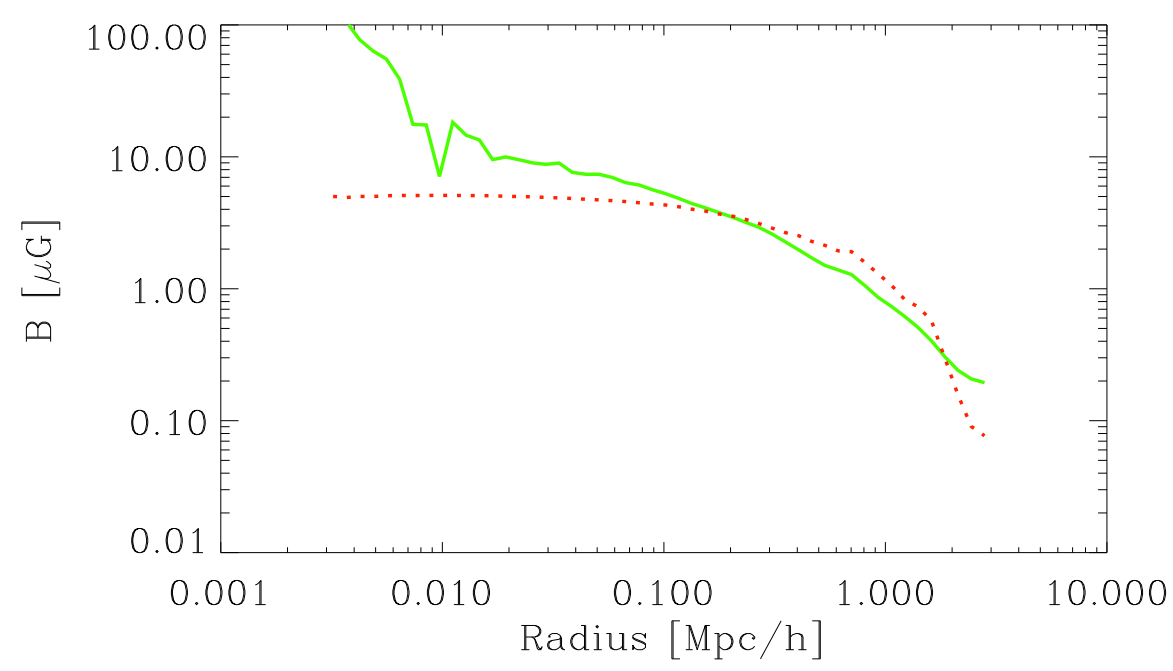

Figure 3: Magnetic Field Profile. The green solid curve is obtained taking the best observational value for a central magnetic field in a coma cluster equal to $4.7 \mu \mathrm{G}$ ( at electron number density of $3.44 \times 10^{-3} \mathrm{~cm}^{-3}$ ) from [8]. The red dotted curve is obtained taking the central magnetic field strength $B_{0}=5.0 \mu \mathrm{G}$ as in the first magnetic field model discussed above.

and

$$
F_{\text {synch }}(x)=x \int_{x}^{\infty} d y K_{5 / 3}(y) \simeq 1.25 x^{\frac{1}{3}} e^{-x}\left(648+x^{2}\right)^{\frac{1}{12}} .
$$

One can obtain the local synchrotron emissivity as a function of electron or positron equilibrium distribution and synchrotron power $P_{\text {synch }}$ as

$$
j_{\text {synch }}(v, r, z)=\int_{m_{e}}^{M_{\chi}} d E\left(\frac{d n_{e^{-}}}{d E}+\frac{d n_{e^{+}}}{d E}\right) P_{\text {synch }}(v, E, r, z) .
$$

The quantity $\frac{d n_{e} \mp}{d E}$ is the electron or positron equilibrium spectra obtained after accounting for the energy loss of electrons through the atmosphere of galaxy cluster, see [4], [7].

Once we obtain the electron equilibrium spectra at each particle position we calculate the local synchrotron emissivity which will allow us to produce the synchrotron emission maps having expressed the flux density within each cube of dimension of $10 \mathrm{kpc}$ :

$$
S_{\text {synch }}(v, z)=\int_{0}^{r} d^{3} r^{\prime} \frac{j_{\text {synch }}\left(v, r^{\prime}, z\right)}{4 \pi D_{L}^{2}},
$$

where $D_{L}$ is the luminosity distance to the halo.

In a DM annihilation process, gamma-ray emission can be produced from pion decay, and inverse Compton (IC) scattering and non-thermal bremsstrahlung processes. In the following we will briefly describe these processes and the formulation used to calculate the gamma-ray flux. 


\section{Pion decay}

When DM annihilation produces neutral pions these unstable particles decay into gamma-rays. The differential flux density from pion decay is then obtained by considering the annihilating DM pairs with mass $M_{\chi}$, their velocity averaged annihilation cross-section $\langle\sigma V\rangle$ and integrating the square of the DM density $\rho_{D M}$ over the volume of the clusters along the line-of-sight divided by surface area of a spherical region of radius $D_{L}$. Hence the differential flux which is number of gamma-ray photons per area per time and per energy is given by

$$
\frac{d F_{\text {gamma }}}{d E}=\frac{<\sigma V>}{2 M_{\chi}^{2}} \frac{d N}{d E_{\gamma}} \int_{0}^{r} \frac{\rho_{D M}^{2} d V}{4 \pi D_{L}^{2}},
$$

where $\frac{d N}{d E_{\gamma}}$ is the initial gamma-ray spectrum.

\section{IC scattering process}

Relativistic electrons produced from the decay of the charged pions, which themselves are the product of the DM annihilation process, scatter the CMB photons and boost them to gamma-rays. The average IC power is given by

$$
P_{I C}\left(E_{\gamma}, \gamma, r\right)=c E_{\gamma} \int_{0}^{\infty} d \varepsilon n(\varepsilon) \sigma\left(E_{\gamma}, \varepsilon, \gamma\right),
$$

where $n(\varepsilon)$ is the black body spectrum of the CMB photons, and $\sigma\left(E_{\gamma}, \varepsilon, \gamma\right)$ is the IC scattering cross-section obtained using

$$
\sigma\left(E_{\gamma}, \varepsilon, \gamma\right)=\frac{3 \sigma_{T}}{4 \varepsilon \gamma^{2}} G\left(q, \Gamma_{e}\right)
$$

where $\sigma_{T}$ is the Thompson cross-section, $\gamma$ is the electron Lorentz factor, and

$$
G\left(q, \Gamma_{e}\right)=\left[2 q \ln q+(1+2 q)(1-q)+\frac{\left(\Gamma_{e} q\right)^{2}(1-q)}{2\left(1+\Gamma_{e} q\right)}\right],
$$

with

$$
\Gamma_{e}=4 \varepsilon \gamma /\left(m c^{2}\right), q=E_{\gamma} /\left[\Gamma_{e}\left(\gamma m c^{2}-E_{\gamma}\right)\right],
$$

The local emissivity is obtained by integrating the product of IC scattering power $P_{I C}\left(E_{\gamma}, \gamma, r\right)$ and electron equilibrium spectrum $N_{e}(\gamma, r)$ across all electron energy as

$$
j_{I C}\left(E_{\gamma}, r\right)=\int_{\gamma \min }^{\gamma \max } d \gamma N_{e}(\gamma, r) P_{I C}\left(E_{\gamma}, \gamma, r\right) .
$$

The flux of the gamma-rays can then be calculated by

$$
S_{I C}\left(E_{\gamma}, z\right)=\int_{0}^{r} d^{3} r^{\prime} \frac{j_{I C}\left(E_{\gamma}, r^{\prime}, z\right)}{4 \pi D_{L}^{2}}
$$




\section{Non-thermal bremsstrahlung radiation}

Non-thermal bremsstrahlung produces gamma-ray radiations when secondary electrons are slowed by the electrostatic field of ions in the intra-cluster medium (ICM) and inter-stellar medium (ISM). The average power of non-thermal bremsstrahlung emission is calculated by considering the number density of ions $n_{j}(r)$

$$
P_{B}\left(E_{\gamma}, \gamma, r\right)=c E_{\gamma} \sum_{j} n_{j}(r) \sigma_{j}\left(E_{\gamma}, E\right),
$$

where the interaction cross-section is given by

$$
\sigma\left(E_{\gamma}, E\right)=\frac{3}{8 \pi} \alpha \sigma_{T}\left[\left[1+\left(1-\frac{E_{\gamma}}{E}\right)^{2}\right] \phi_{1}-\frac{2}{3}\left(1-\frac{E_{\gamma}}{E}\right) \phi_{2}\right],
$$

with

$$
\begin{gathered}
\phi_{u}=4\left[\ln \left[\frac{2 E}{m c^{2}}\left(\frac{E-E_{\gamma}}{E_{\gamma}}\right)\right]-\frac{1}{2}\right] \\
j_{B}\left(E_{\gamma}, r\right)=\int_{\gamma \min }^{\gamma \max } d \gamma N_{e}(\gamma, r) P_{B}\left(E_{\gamma}, \gamma, r\right) .
\end{gathered}
$$

In a similar way to IC scattering the local emissivity $j_{B}\left(E_{\gamma}, r\right)$ is obtained which will then be used in

$$
S_{B}\left(E_{\gamma}, z\right)=\int_{0}^{r} d^{3} r^{\prime} \frac{j_{B}\left(E_{\gamma}, r^{\prime}, z\right)}{4 \pi D_{L}^{2}} .
$$

to calculate the gamma-ray flux from non-thermal bremsstrahlung process.

\section{Results and Discussion}

In the following we present the results that we have obtained for synchrotron emission from DM annihilation processes discussed in section 2.2 using the two magnetic field models (Model A and Model B) discussed in section 2.1. The emission maps are based on the MUSIC-2 dataset [5], a high-resolution cosmological simulation containing DM, gas, stars and feedback processes. We compute the expected flux for synchrotron emission processes from DM annihilation by dividing a cluster of dimension $2 \mathrm{Mpc}$ into cubes of size $10 \mathrm{kpc}$. We based our studies of radio emission from DM annihilation processes by taking neutralinos to be primarily and dominantly composed of $b \bar{b}$ species and/or light and intermediate DM mass models as in [10]. We consider neutralino masses $M_{\chi}$ of 35 and $60 \mathrm{GeV}$, and we take the velocity averaged annihilation cross-section, $\langle\sigma V\rangle$, of $1.0 \times 10^{-26} \mathrm{~cm}^{3} \mathrm{~s}^{-1}$ from Fermi upper limit.

\subsection{Synchrotron emission maps and flux in galaxy cluster}

The synchrotron emission maps in Fig. 4 and 5 show the radio flux obtained at $110 \mathrm{MHz}$ and 1.4 GHz. These two frequencies were chosen to represent the SKA-low and SKA-mid frequencies [11]. The synchrotron emission maps in both Fig. 4 and 5 are obtained using magnetic field model A for neutralino mass of $M_{\chi}=60 \mathrm{GeV}$. The central region of the cluster shows a strong radio flux because of its high DM density. The synchrotron emission flux calculation is able to resolve the contribution from DM dominated regions in the cluster, thus halos and substructures that are 
residing off center are also be clearly visible in the maps. The maps also show the presence of two main halos in the cluster which are approximately $0.5 \mathrm{Mpc}$ apart. Comparison between Fig. 4 and 5 (i.e maps that are produced at $110 \mathrm{MHz}$ and $1.4 \mathrm{GHz}$ frequencies, respectively) do not show any noticeable difference on the background structure of the emission apart from the difference in the output flux at the two frequencies. Point-by-point comparison between the two radio emission maps show flux values at $110 \mathrm{MHz}$ (Fig. 4) varies between 9 to 80 times that of flux at $1.4 \mathrm{GHz}$ (Fig. 5).

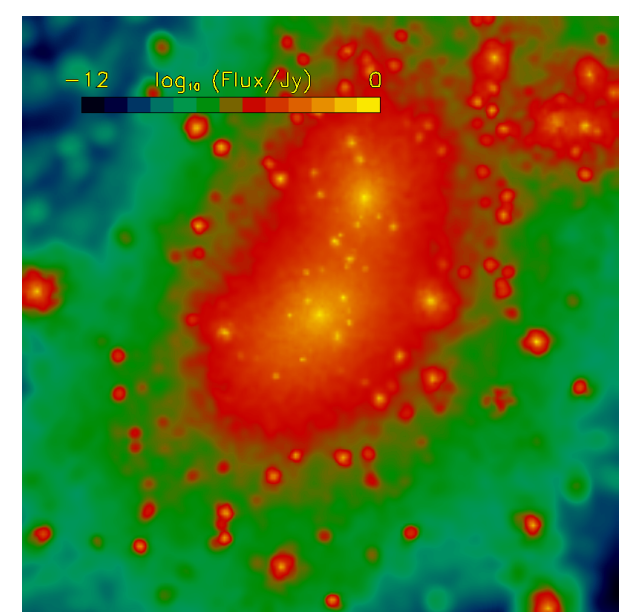

Figure 4: Radio emission map at $110 \mathrm{MHz}$ using magnetic field of Model A for DM model $60 \mathrm{GeV}$.

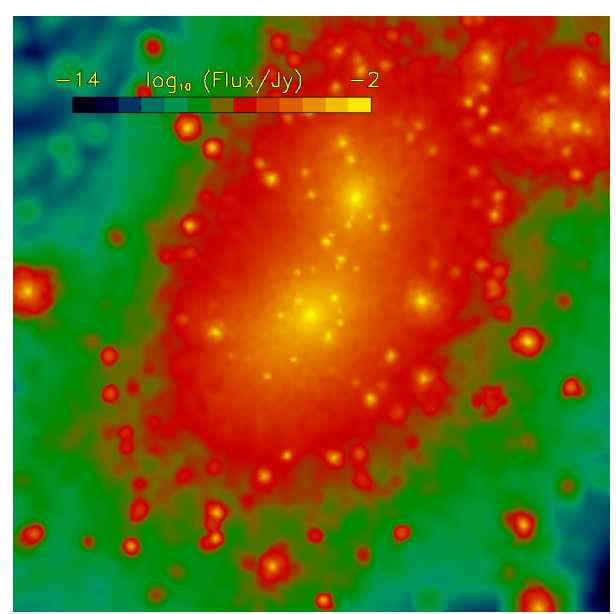

Figure 5: Radio emission map at $1.4 \mathrm{GHz}$ using magnetic field of Model A for DM model $60 \mathrm{GeV}$.

Figure 6 shows the radial variation of the flux with radius. It is obtained from the flux values in the maps of Fig. 4 and 5, by picking the flux values starting from the center of the map and moving to the bottom of the map. The sharp flux variations shown at large radii indicate that the flux from substructures are dominant in the outer parts the cluster. The two solid curves in the top part of the figure are obtained for frequency of $110 \mathrm{MHz}$, and the bottom ones in the dotted curves are for frequency of $1.4 \mathrm{GHz}$. The flux profile obtained with the two magnetic field models are indicated as Model A in red color and Model B in black color, respectively. In the inner region of the cluster the flux values based on Model A are slightly lower than that based on Model B and vice versa in the outer region. This is due to the magnetic field values of Model B which have higher values in the inner region as shown in Fig. 3. Moreover, the flatter regions which show the cuts that are observed around the end of the curves, or central distance of $\sim 700 \mathrm{kpc}$, arise due to our subtraction of the SKA sensitivity (from [11]) of the flux values per beam at the two frequencies.

In order to explore better the difference in the emission maps that are obtained using the two magnetic field models, we also produced maps shown in Figs. 7 and 8 which are obtained using magnetic field of Model B and neutralino mass of $60 \mathrm{GeV}$. Point-by-point comparison between the two radio emission maps show flux values at $110 \mathrm{MHz}$ (Fig. 7) varies between 6 and 38 times that of flux at $1.4 \mathrm{GHz}$ (Fig. 8).

For the neutralino mass of $60 \mathrm{GeV}$ used, we have mentioned above that a point-by-point comparison between the two radio emission maps that are obtained using magnetic field model A shows 




Figure 6: Flux profiles at frequency $110 \mathrm{MHz}$, solid curves, and $1.4 \mathrm{GHz}$, dotted curves. The mass of neutralino used is $60 \mathrm{GeV}$ along with magnetic field Model A indicated in red color and Model $\mathrm{B}$ in black.

flux at $110 \mathrm{MHz}$ (Fig. 4) varies between 9 and 80 times that of flux at $1.4 \mathrm{GHz}$ (Fig. 5). On the other hand the flux values obtained using magnetic field Model B (Figs. 7 and 8) varies with smaller values between 6 and 38. Hence in Fig. 9 we see a flatter appearance of the flux density curve for magnetic field of Model B (cyan color) than that of Model A (red color).

Similarly we have considered the case of neutralino mass of $35 \mathrm{GeV}$ for in our point-by-point comparisons. We have obtained the flux values in the emission maps of Figs. 38 and 39, which are obtained using magnetic field of Model A for the two frequencies of 110 and $1400 \mathrm{MHz}$, varies between 12 to 433, respectively. Whereas we obtained the flux variations for the emission maps which uses magnetic field Model B (Figs. 40 and 41) at the two frequencies of 110 and $1400 \mathrm{MHz}$, varies at smaller ranges of 7 to 77 . To see what these variations obtained from point-by-point comparisons translates into, we have calculated the spectral indexes of this four cases. Table 1 provides these results of our comparison of the spectral index for synchrotron flux densities that are calculated taking 110 and $1400 \mathrm{MHz}$ frequencies for which we showed their emission maps in the above discussions. We found the highest negative value for spectral index of $35 \mathrm{GeV}$ neutralino mass and magnetic field Model A as compared to all the four cases given in table 1. 


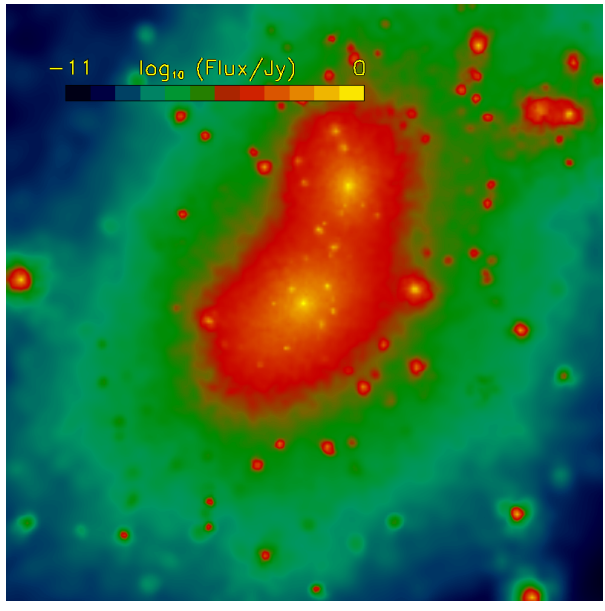

Figure 7: Radio emission map at $110 \mathrm{MHz}$ using magnetic field of Model B for DM model $60 \mathrm{GeV}$.

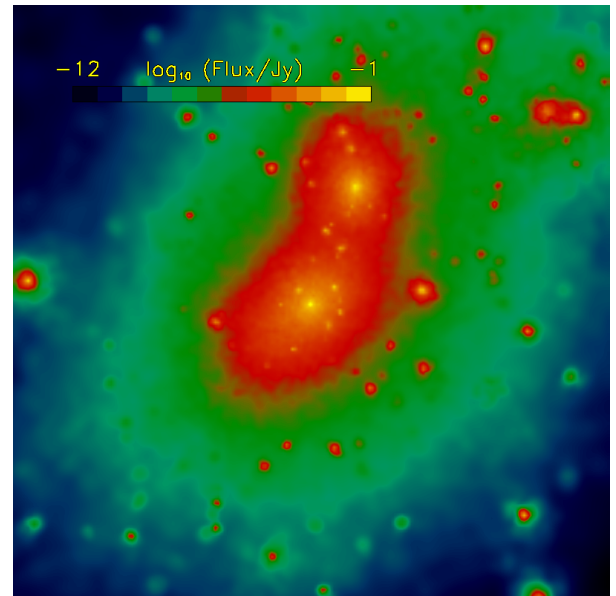

Figure 8: Radio emission map at $1.4 \mathrm{GHz}$ using magnetic field of Model B for DM model $60 \mathrm{GeV}$.

\begin{tabular}{|c|l|l|}
\hline $\begin{array}{l}\text { Neutralino Mass } \\
{[\mathrm{GeV}]}\end{array}$ & $\begin{array}{l}\text { Magnetic Field } \\
\text { Models }\end{array}$ & Spectral Index \\
\hline 35 & Model A & -1.16 \\
\hline 35 & Model B & -0.98 \\
\hline 60 & Model A & -1.05 \\
\hline 60 & Model B & -0.91 \\
\hline
\end{tabular}

Table 1: Comparison of the spectral index for radio flux densities using neutralino annihilation final product of $b \bar{b}$ species.

Figure 9 shows the flux density from synchrotron emission in comparison with the observational data of the Coma cluster (blue data points) [12], taking mass of neutralino $60 \mathrm{GeV}$, dotted curves and $35 \mathrm{GeV}$, solid curves. Magnetic field model used are indicated in cyan and red color for Model A and B, respectively. The fact that these flux densities show a very good agreement with the observational result of the Coma cluster data without invoking a boost factor from DM substructures shows that the distribution of sub-structures is described well in the simulations which boosted the signals from DM annihilation processes exactly to the level of observed flux.

As our result of the spectral index reflects in table 1 we see a steeper profile among the four case for magnetic field Model A and $35 \mathrm{GeV}$ neutralino mass. Hence the best fit to the observational result of [12] is obtained taking neutralino mass of $35 \mathrm{GeV}$ and magnetic field model A. However, we have also observed the flux density for the radio halo spectrum obtained for neutralino masses of both $M_{\chi}=60$ and $35 \mathrm{GeV}$ as in Fig. 9 are flatter than the measured result of [12] at higher frequency ranges. A more recent paper by [13] has shown that the best agreement in the overall flux density spectrum is obtained using smaller values of neutralino mass of $9 \mathrm{GeV}$ and magnetic field of Model B.

In order to understand why we see a flatter spectrum when we use magnetic filed Model B, we constrain the magnetic field values of Model B. That is the flux densities for magnetic field 


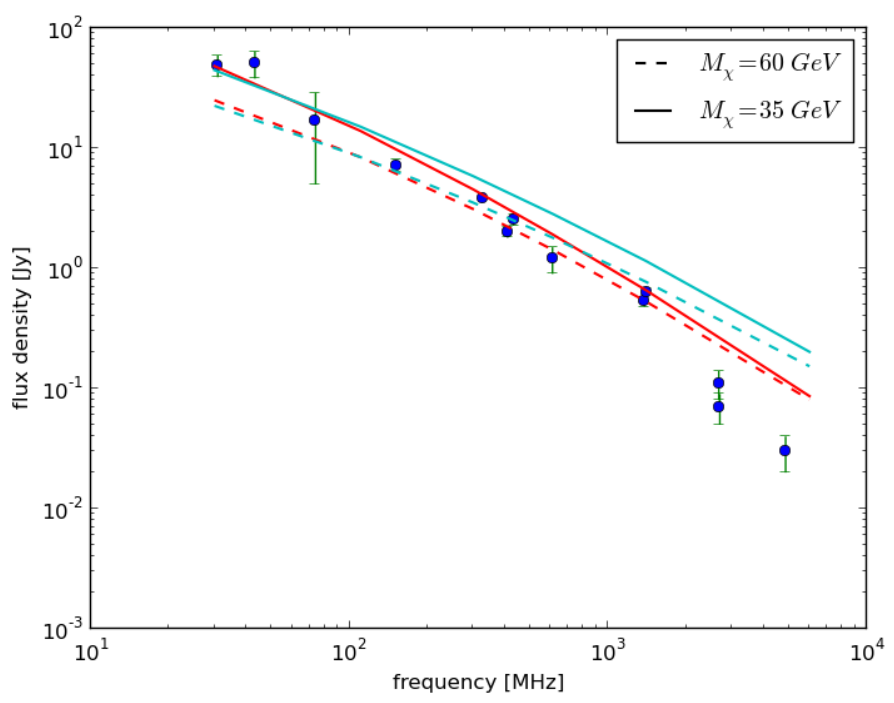

Figure 9: Our estimates for the flux density from synchrotron emission are compared with observations of the Coma cluster from [12] for neutralino masses of $60 \mathrm{GeV}$ (dotted curves) and $35 \mathrm{GeV}$ (solid curves). Magnetic field model used are Model A, in red and Model B in cyan color.

Model B constrained are obtained by setting the magnetic field values which are above $7.0 \mu \mathrm{G}$ to $7.0 \mu \mathrm{G}$. As shown in Fig. 10 the flux densities of Model B constrained indicated in red curve shows a steeper spectrum than that of Model B which is indicated in cyan color. We find that the main effect of the high magnetic field is an enhancement of the radio flux density from the central region of the cluster. However, this enhancement can be reduced by constraining the magnetic field as shown by using Model B constrained in the red curve. This is resulted as some values of the radio flux densities at higher frequencies get shifted to the values at lower frequencies. Over all this shows us both magnetic field models, Model A and Model B are able to reproduce the radio flux spectrum. It is worth mentioning why we have not constrained the magnetic field according to the " $\beta$-model". The reason is that for our calculation of magnetic field using the electron density from the MUSIC-2 simulation we wanted to see it following the gas density or equivalently the electron number density. However, the $\beta$-model uses the gas densities that are constrained as

$$
n_{e l}(r)=n_{e l, 0}\left(1+\left(r / r_{c}\right)^{2}\right)^{-\frac{3}{2} \beta}
$$

where $\beta$ is a constant, $n_{e l, 0}$ is the central gas density, $r$ is the radial distance from cluster center and $r_{c}$ the cluster core radius.

It is worth mentioning that the simulated clusters we have used for our studies of DM annihilation processes in galaxy clusters, from the MUSIC-2 cluster simulations, in general show higher values of gas densities in their central regions as they suffer from cooling. Thus they give larger values of magnetic field based on the formulation of equation 2.3. Despite this drawback the magnetic field calculations based on electron densities of magnetic field Model B show a fair agreement in large part of the cluster volume. In addition to this the formulation in equation 2.3, 
unlike the electron number densities constrained as in equation 3.1, will allow us to apply our magnetic field calculations for all the 282 clusters we studied. This simple modification we made thus has the following advantages: (i) it is able to give magnetic field values at each grid as compared to the constrained model which follows a radial variations which assumes spherical symmetry in the cluster, (ii) it reduces model dependencies and, (iii) it allows a robust study of both magnetic field and emission processes from DM annihilation processes.

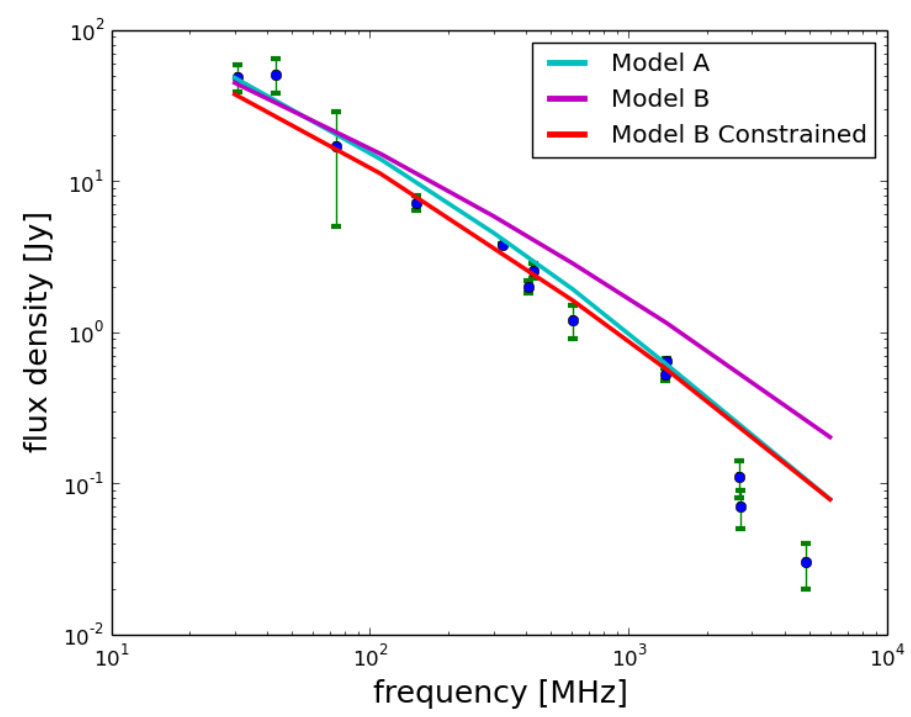

Figure 10: Flux density of synchrotron emission process for neutralino mass of $35 \mathrm{GeV}$ (solid curves) is compared with observational result of Coma $\mathrm{C}$ data (blue data points) from [12]. Magnetic field models used are indicated in cyan, magenta and red for Model A, Model B, and Model B constrained, respectively.

\subsection{Gamma-ray emission maps and flux in galaxy cluster}

In this section we will present some of the results we have obtained for gamma-ray emission from the DM annihilation processes in galaxy clusters. We have produced emission maps and calculated the gamma-ray flux by considering all important processes: pion decay, inverse compton (IC) scattering and Bremsstrahlung radiation processes which will produce gamma-ray emission from DM annihilation processes. Based on the result in Fig. 9 we chose neutralinos mass of $M_{\chi}$ of $35 \mathrm{GeV}$ and composition of $b \bar{b}$ which is the best fitting DM species and mass for DM annihilation models as it has also been suggested by the data of cosmic ray antiproton spectrum obtained by PAMELA [14] which is in agreement with the result of [13]. We use neutralino annihilation velocity averaged cross-section of $1.0 \times 10^{-26} \mathrm{~cm}^{3} \mathrm{~s}^{-1}$ for our calculation.

Figure 11 and 12 show the gamma-ray emission maps obtained from non-thermal Bremsstrahlung process at a photon energy of $100 \mathrm{MeV}$. These two gamma-ray emission maps are obtained using a varying electron density given in Eqn. 2.4 from reference [5] and a constant average electron density of $\mathrm{n}_{\mathrm{e} 0}=3.4 \times 10^{-3} \mathrm{~cm}^{-3}$, respectively. We have used this central value of $\mathrm{n}_{\mathrm{e} 0}$ from the 
Coma cluster only for the purpose of comparison. The gamma-ray flux emanating from the dense central point of the cluster, shown in bright color of Fig. 11, is 10 times larger than that of Fig. 12 , which results from the non-thermal bremsstrahlung power dependence on the electron number density that has its peak in the center of cluster. In Fig. 12 we also see that a wide variation (11 orders of magnitude $10^{-24}$ to $4 \times 10^{-13}$ ) between lower and higher values of differential gamma-ray flux in the color bar.

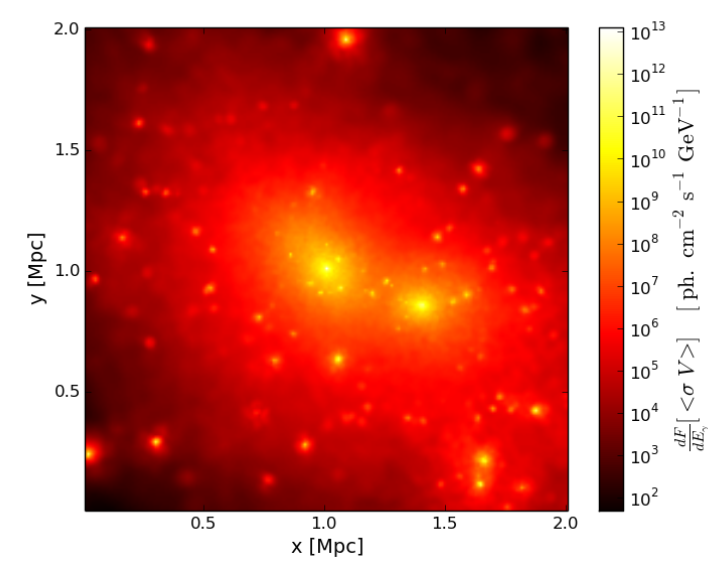

Figure 11: Gamma-ray emission from nonthermal bremsstrahlung process at $100 \mathrm{MeV}$ using radially varying thermal electron density from reference [5] in units of $\langle\sigma V\rangle$.

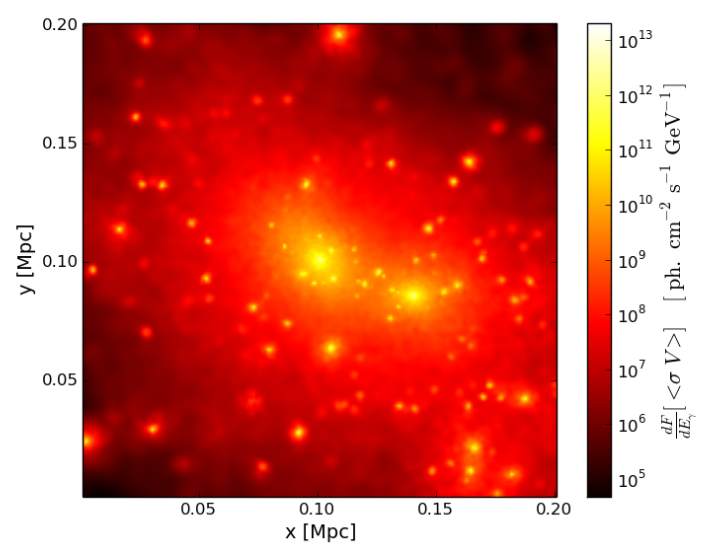

Figure 13: Gamma-ray emission from pion decay processes at $100 \mathrm{MeV}$ in units of $\langle\sigma V\rangle$.



Figure 12: Gamma-ray emission from non-thermal bremsstrahlung process at $100 \mathrm{MeV}$ using a constant average electron density as found in the Coma cluster.

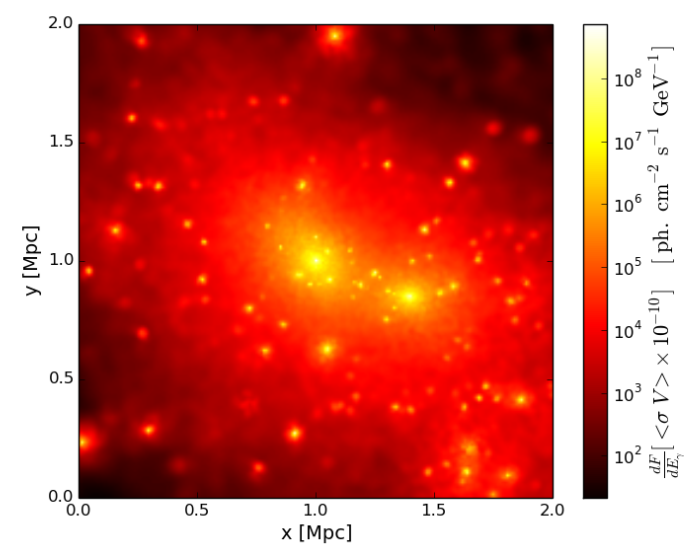

Figure 14: Gamma-ray emission from inverse compton scattering process at $100 \mathrm{MeV}$ in unit of $10^{-10}\langle\sigma V\rangle$.

The gamma-ray emission map obtained from pion decay and IC scattering processes at a photon energy of $100 \mathrm{MeV}$ are shown in Figs. 13 and 14 respectively. The magnitude of the flux obtained from IC scattering processes is 15 orders of magnitude less than that of pion decay at 
gamma-ray photon energy of $100 \mathrm{MeV}$. It is expected that for neutralino mass used, $35 \mathrm{GeV}$, the IC scattering contribution is negligible below photon energy of $10 \mathrm{MeV}$.

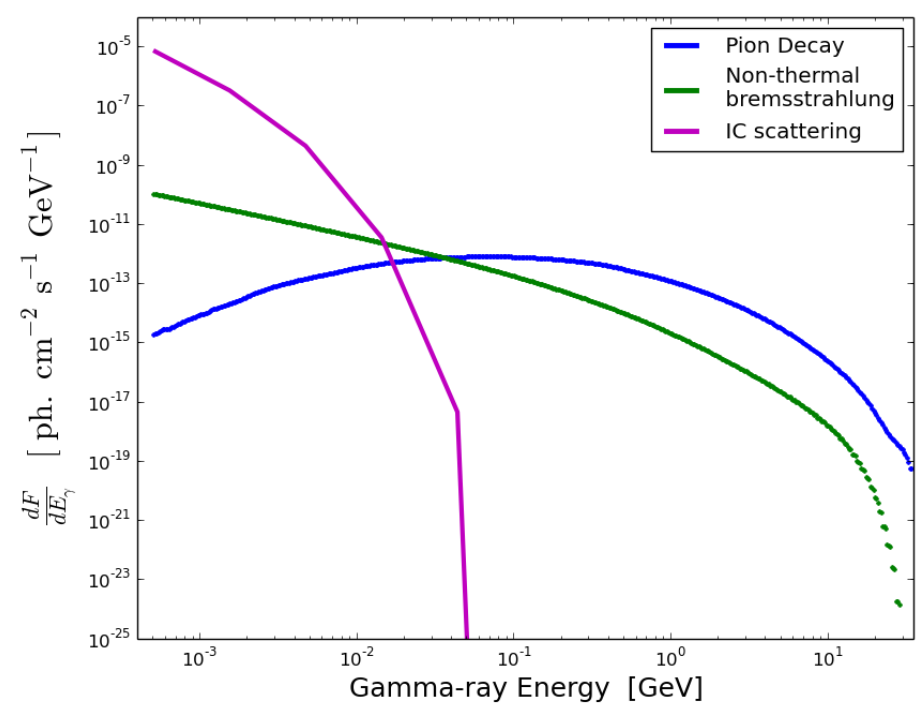

Figure 15: Differential gamma-ray flux at frequencies above $100 \mathrm{MeV}$. The gamma-ray flux from pion decay, non-thermal bremsstrahlung and IC scattering processes is given in the plot by blue, green and magenta colors.

In Fig. 15 we show the differential gamma-ray flux densities obtained from pion decay, nonthermal bremsstrahlung and IC scattering processes, respectively. The frequencies range on the $\mathrm{x}$-axis starts at mass of electron $0.511 \mathrm{MeV}$ to a complete annihilation/decay of neutralino mass of $35 \mathrm{GeV}$ used. The contribution of the gamma-ray flux from IC dominates at lower energies. Non-thermal bremsstrahlung is most prominent at intermediate energies. At about $35 \mathrm{MeV}$ pion decay takes over until it decays completely at the mass of neutralino $35 \mathrm{GeV}$. The shape of spectra we obtained here for neutralino mass of $35 \mathrm{GeV}$ and composition $b \bar{b}$ is in agreement with the recent work in reference [13] of a similar (intermediate range) neutralino mass of $43 \mathrm{GeV}$ composition $b \bar{b}$.

In table 2 we provide comparison of gamma-ray integrated flux above $100 \mathrm{MeV}, 1 \mathrm{GeV}$, and $10 \mathrm{GeV}$ with the Fermi upper limits [15]. Our integrated gamma-ray flux values are roughly 4, 3 and 4 orders of magnitude less than the Fermi upper limits for gamma-ray energies above $0.1,1$ and $10 \mathrm{GeV}$, respectively.

\begin{tabular}{|l|l|c|}
\hline $\begin{array}{l}\text { Band } \\
\mathrm{GeV}\end{array}$ & $\begin{array}{l}\text { Upper limit } \\
\mathrm{cm}^{-2} \mathrm{~s}^{-1}\end{array}$ & $\begin{array}{l}\text { Flux }(35 \mathrm{GeV} \text { model }) \\
\mathrm{cm}^{-2} \mathrm{~s}^{-1}\end{array}$ \\
\hline$>0.1$ & $4.2 \times 10^{-9}$ & $4.0 \times 10^{-13}$ \\
\hline$>1$ & $1.13 \times 10^{-10}$ & $1.02 \times 10^{-13}$ \\
\hline$>10$ & $6.0 \times 10^{-12}$ & $5.72 \times 10^{-16}$ \\
\hline
\end{tabular}


Table 2: Gamma-ray upper limits set by Fermi for energies above 0.1, 1 and $10 \mathrm{GeV}$ (from [15]) and the comparison with our flux calculation using neutralino mass of $35 \mathrm{GeV}$ and $b \bar{b}$ species.

\subsection{The search for DM dominated sub-halos}

When two galaxy clusters undergo a merger they will leave the DM structure physically separated from the gas and stars [16]. The DM structure resides in the outer part of the cluster making it a suitable place to look for the DM annihilation signals. Another promising place to study the DM annihilation emission process is to focus on DM halos which contains a very small proportion of gas and stars or DM halos that are entirely devoid of gas and stars. Here within, we will consider the latter option to study an emission process from self annihilating DM particles.

The Rockstar halo finder provides an efficient method to identify halos in a seven-dimension phase space (position, velocity, time) [17]. Rockstar can provide different halo properties, the halo masses at several radius, halo radius, halo center position and velocity, halo spin and angular momentum, etc.

A very promising place to look for an emission from a DM annihilation process is to isolate those sub-halos containing a small fraction of gas and stellar content, which are dominated by their DM compositions. Using the mass-to-light ratio, $\mathrm{M} / \mathrm{L}$, we isolate sub-halos in the region of the emission map of $2 \mathrm{Mpc}$ diameter we discussed previously. Figure 16 shows the DM sub-halos over plotted in filled black dots on the top of the synchrotron emission.

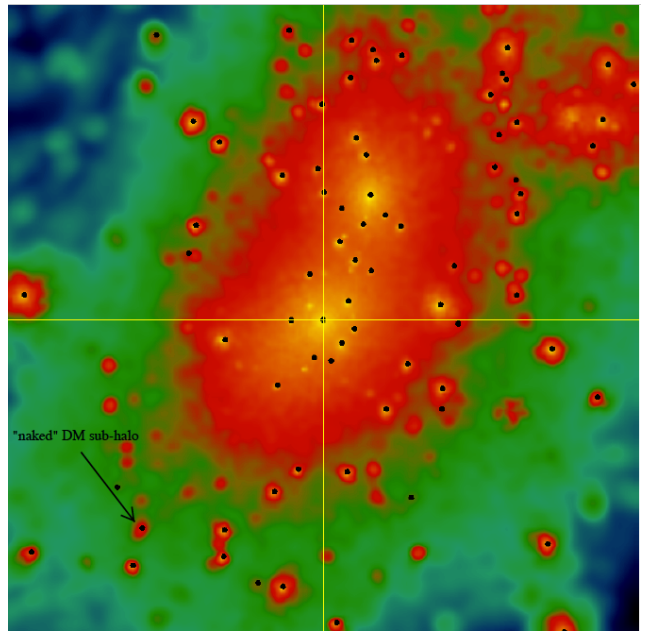

Figure 16: Identifying the DM sub-halos filled black dots on the top of the synchrotron emission.

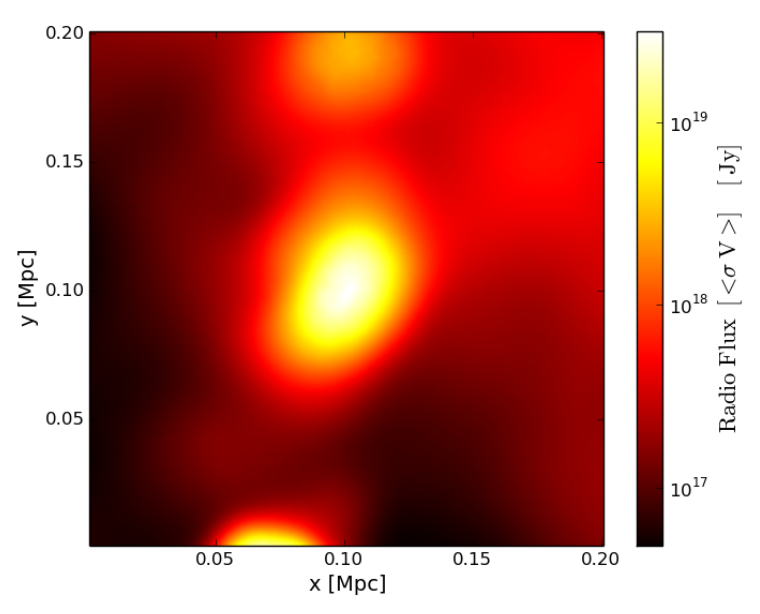

Figure 17: Synchrotron emission map at 110 MHz of DM sub-halo 1, the DM dominated sub-halo indicated by the arrow in the map on the right.

Having high $\mathrm{M} / \mathrm{L}$ is not sufficient as sometimes a tidal disruption produces a high $\mathrm{M} / \mathrm{L}$. Looking into the particle distribution within a cubic section is therefore important to assess if the subhalo displays an enhanced DM distribution. Having gone through the halos in Fig. 16, the sub-halo within the lower-left quadrant indicated by the arrow in Fig. 16 stands out as a best candidate to study synchrotron emission from the DM annihilation process. This sub-halo has a M/L of 30.55 
which shows an enhancement in the DM distribution and a gas fraction of $\sim 0.5 \%$ and stellar fraction of $\sim 3 \%$.

For neutralino mass, $M_{\chi}, 35 \mathrm{GeV}$ and DM annihilation cross-section, $\langle\sigma V\rangle$, of $1.0 \times 10^{26}$ $\mathrm{cm}^{3} \mathrm{~s}^{-1}$ and magnetic field calculated using the electron density at each particle position using the expression given in equation 2.3 of [8] we produce a synchrotron emission map for a single sub-halo that is analyzed above. The maps are obtained by centering at the center of the halo with diameter of $200 \mathrm{kpc}$ and resolution of $\mathrm{L}_{\mathrm{x}}=\mathrm{L}_{\mathrm{y}}=1 \mathrm{kpc}$, and $\mathrm{L}_{\mathrm{z}}=10 \mathrm{kpc}$.

Henceforth we refer to the above discussed single sub-halo as DM sub-halo 1 whose some of its properties are listed in Tabs. 3 and 4. The synchrotron emission map at frequency of 110 $\mathrm{MHz}$ and the flux density for DM sub-halo 1 are shown in Figs. 17 and 18, respectively. The obtained values of the flux density for DM sub-halo 1 will be shown latter to be in the range of SKA sensitivity limit (see last part of Section 4). The comparison of shape and slope with the simulated Coma like cluster and the Coma cluster data of [12], is given in Fig. 19. The spectral shape for DM sub-halo 1 (red curve), which is shown with the scaled comparison, is steeper than its host cluster and seem to match the Coma cluster observational data (blue data points) over wide wavelength range as compared to its host cluster (cyan curve).


Figure 18: Radio flux density of DM sub-halo 1 Figure 19: Slope comparison of the flux density compared with the host cluster (Cluster 280) and observational data of the Coma cluster for synchrotron emission of DM sub-halo 1 with the host cluster and observational data of the (blue data points). Coma cluster (blue data points).

From all the 282 MUSIC- 2 clusters we have obtained a total of 466,621 sub-halos of which 132,614 are with in $2.5 \mathrm{R}_{200 \text { crit }}$ of their host cluster, an approximate distance where we can obtain resolved particles. A linear plot of the mass-to-light ratio of all these halos plotted against their relative distance to their respective clusters ( i.e., ratio of the sub-halo distance by the cluster radius they belong to $\left(\mathrm{R} / \mathrm{R}_{200 \text { crit }}\right)$ ) is shown in Fig. 20. ${ }^{1}$ We observe some behavior of grouping of sub-halos with high $\mathrm{M} / \mathrm{L}(\sim 360 \pm 70)$ as indicated in the shaded region around $\mathrm{R} \sim 0.5 \times \mathrm{R}_{200 \text { crit }}$ of their host clusters.

\footnotetext{
${ }^{1} \mathrm{R}_{200 \text { crit }}$ is the cluster radius obtained at the over-density of 200 times the critical density.
} 
The scatter plot given in Fig. 21 shows the mass-to-light ratio of the sub-halos plotted against the stellar mass within the sub-halos. We see that sub-halos which have single stars and less gas fraction are seen to be located on the left upper part of the plot. However, to choose the best candidates of "naked" DM sub-halos which will allow us to study the multi-wavelength emission from DM annihilation processes, we first need to look closer into their particle distribution and identify if there is an enhancement in the DM particle distribution. More importantly we also have to check on the gas and star content of the sub-halos. Following these procedure we have identified large number of high M/L DM sub-halos. In section 4 we will provide some of the result of the multi-wavelength emissions produced in these objects.

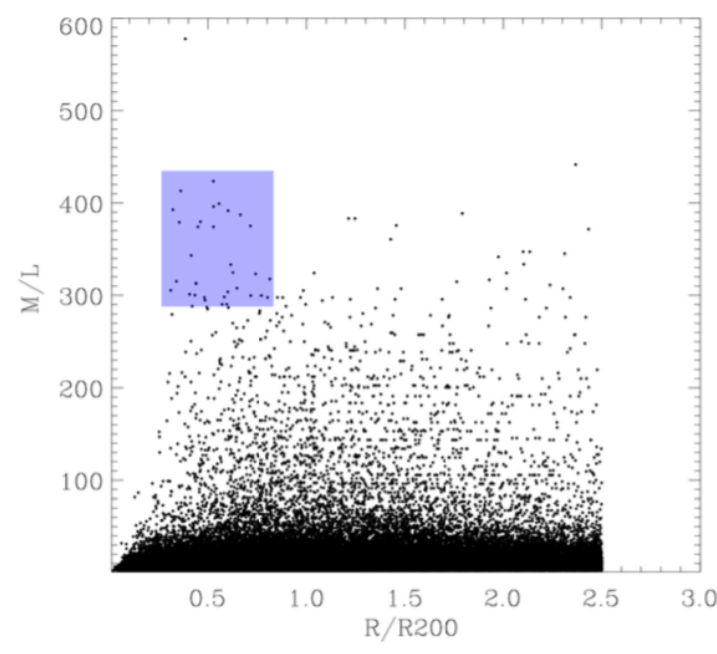

Figure 20: Scatter plot of the DM sub-halos based on $\mathrm{M} / \mathrm{L}$ and the ratio of their radial distance $(\mathrm{R})$ to radius of their clusters $\left(\mathrm{R} 200 \equiv \mathrm{R}_{200 \text { crit }}\right)$.



Figure 21: Scatter plot of the DM sub-halos based on their $\mathrm{M} / \mathrm{L}$ and stellar content.

As was pointed out earlier a clean signal from DM sub-halos requires to locate high M/L DM sub-halos which contain less baryons (gas and stars).In this regard it is important to find out where we have a relatively high chance of finding high M/L DM sub-halos. We will study these using the following scatter plots and histograms. The scatter plot in Fig. 22 and 23 gives the relative distance of DM sub-halos in the clusters they belong to identified based on $\mathrm{M} / \mathrm{L}$ and stellar content. We have indicated the DM sub-halos which have M/L above 50 and 100 by red color and among all DM sub-halos identified with in radial distance of $2.5 \mathrm{R}_{200 \text { crit }}$ in Fig. 22 and 23, respectively. These two plots show that the high $\mathrm{M} / \mathrm{L}$ DM sub-halos are rarely found in the inner part of clusters.

In Fig. 24 we show the probability density distribution $\mathrm{p}(\mathrm{r})$ of sub-halos versus their relative location in their cluster $r \equiv R / R_{200 \text { crit }}$ (the ratio of the distance from the center of the cluster to the radius of the cluster at an overdensity 200 times the critical density of the universe). DM sub-halos with $\mathrm{M} / \mathrm{L}$ above 1,50 and 100 are indicated in blue, green and red, respectively. The standard error for a count of the DM sub-halos is also indicated by the vertical error bars. From the histogram we see that the probability of finding DM sub-halos with M/L above 100 (red curve) in the inner region is low as it shows a sharp decline in the lower values of r. From the peak of the histogram 


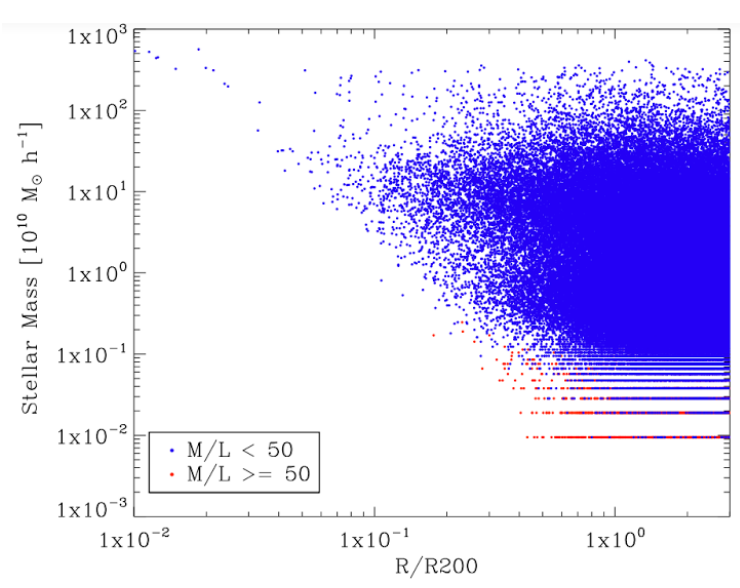

Figure 22: Scatter plot of DM sub-halos based on their stellar content at M/L of 50 .

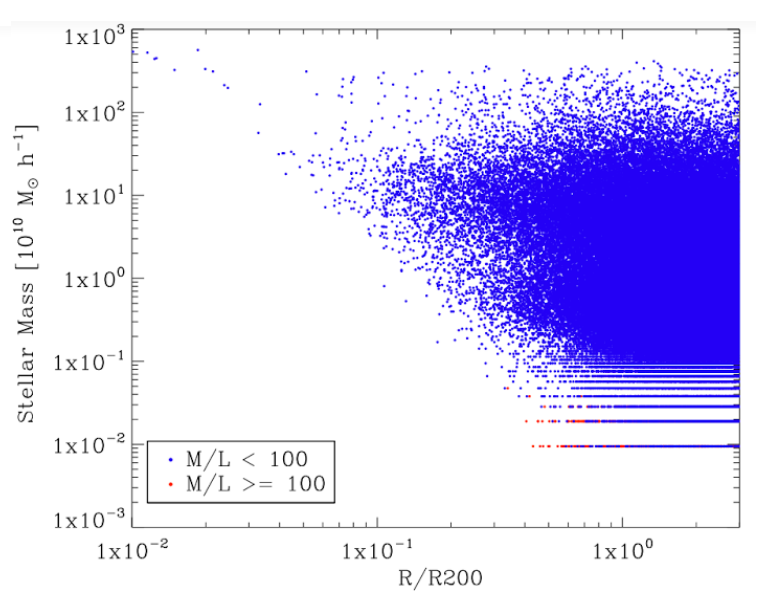

Figure 23: Scatter plot of DM sub-halos based on their stellar content at M/L of 100 .

we deduce that while some high $\mathrm{M} / \mathrm{L}$ DM sub-halos exist in the outer part they are seen to be more populated around $\mathrm{r} \sim \mathrm{R}_{200 \text { crit }}$.

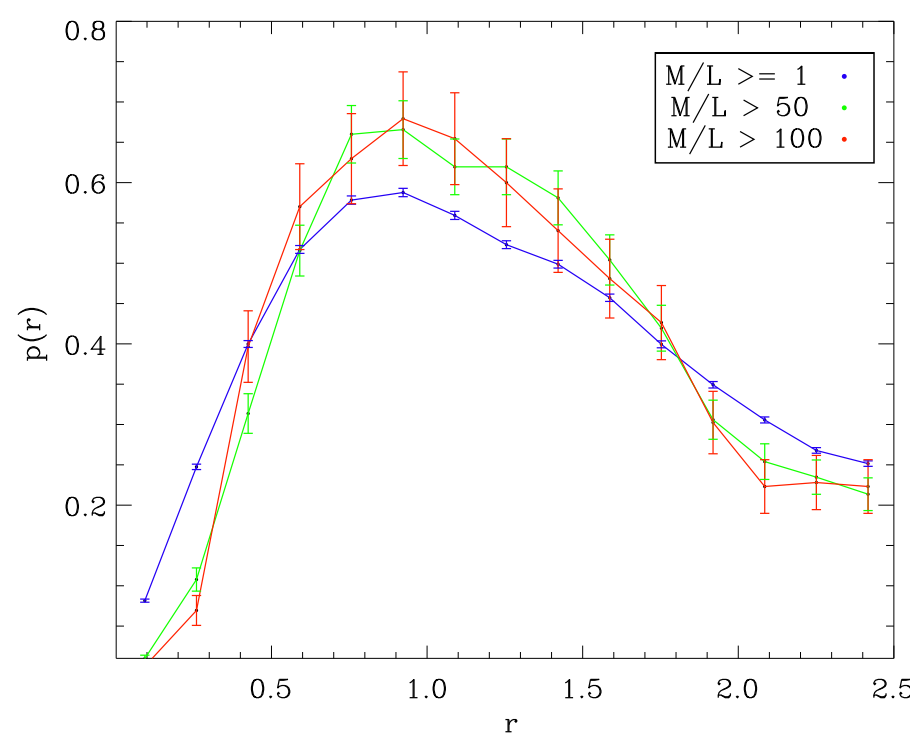

Figure 24: The probability density distribution $\mathrm{p}(\mathrm{r})$ vs cluster centric distances of sub-halos $r$ based on their M/L. DM sub-halos with M/L above 1, 50 and 100 are shown in blue, green and red, respectively.

\section{High $M / L$ sub-halos and the radio emission from DM annihilation processes.}

Here we present and discuss some of the results we obtained for density square maps, the Synchrotron/radio emission processes using radio maps and flux densities for two representative 
samples of high M/L DM sub-halos, whose properties are listed in Tabs. 3 and 4. These calculation were made for neutralino mass of $\mathrm{M}_{\chi}, 35 \mathrm{GeV}$ which is chosen based on our result in section 3.1 and velocity averaged DM annihilation cross-section, $\langle\sigma V\rangle$, of $1.0 \times 10^{-26} \mathrm{~cm}^{3} \mathrm{~s}^{-1}$ from Fermi upper limit.

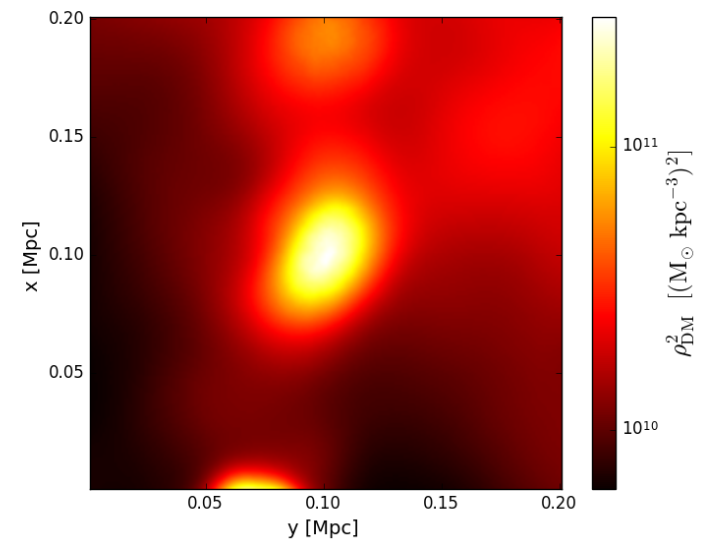

Figure 25: Map of the square of the DM density for DM sub-halo 1 integrated along the line-of-sight.



Figure 27: Map of the square of the DM density for DM sub-halo 2 integrated along the line-of-sight.

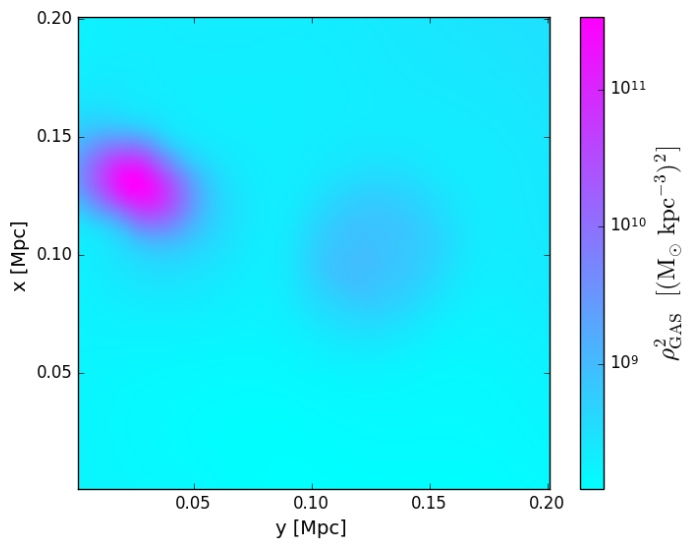

Figure 26: Map of the square of the gas density for DM sub-halo 1 integrated along the line-of-sight.

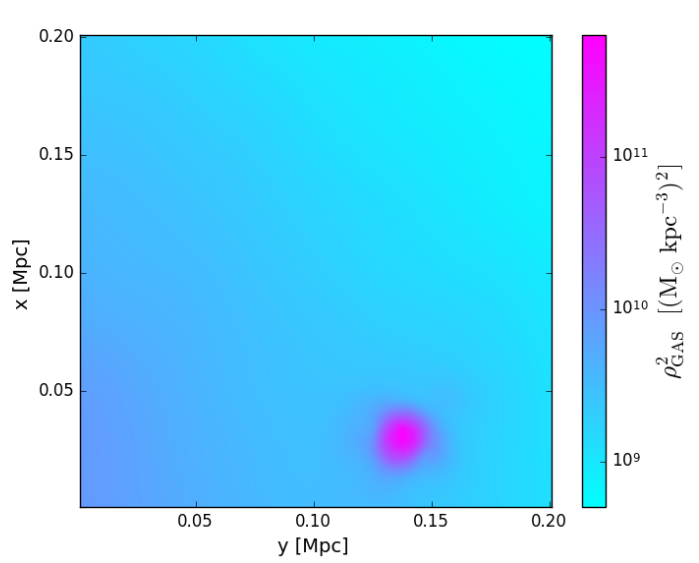

Figure 28: Map of the square of the gas density for DM sub-halo 2 integrated along the line-of-sight.

In Figs. 25 and 26 we show the map of the square of the DM and gas densities of the DM sub-halo 1, respectively. We see a clear enhanced DM concentration around the center of the map of the square of the DM of the DM sub-halo 1, as the bright color in the map reflects. We also see that these regions are offset with respect to enhanced regions on gas density map of Fig. 26, which is crucial to reduce or avoid emission contamination from baryonic matter.

The second DM sub-halo we consider is DM sub-halo 2 whose DM and gas density square maps are given in Figs. 27 and 28. There are three dense DM structures located in different regions 
with respect to the gas density enhancement regions. It also shows higher values of DM densities compared to DM sub-halo 1.

In Figs. 29 and 30 we show the synchrotron emission maps obtained at 110 for DM sub-halo 1 and 2 in units of $\langle\sigma V\rangle[\mathrm{Jy}]$ whose DM and gas density square maps are provided in Figs. 25, 27 and 26, 28, respectively. The synchrotron emission maps show similar features of to the DM maps as the DM density square values are the locations where emission takes place.

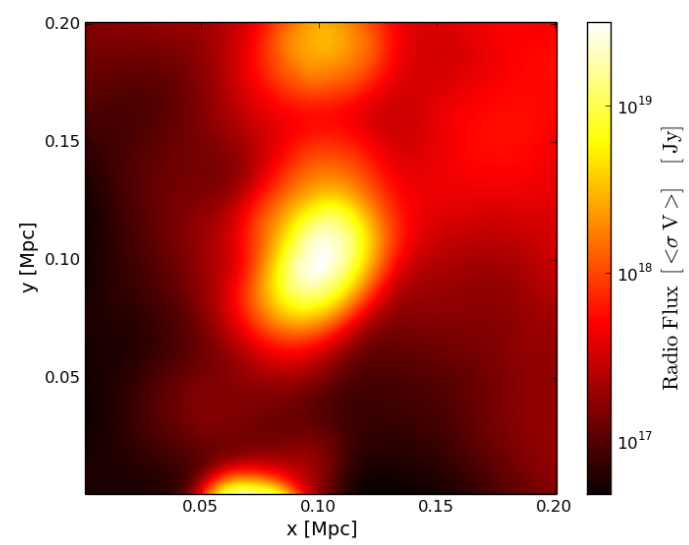

Figure 29: Synchrotron emission map of DM sub-halo 1 at $110 \mathrm{MHz}$.

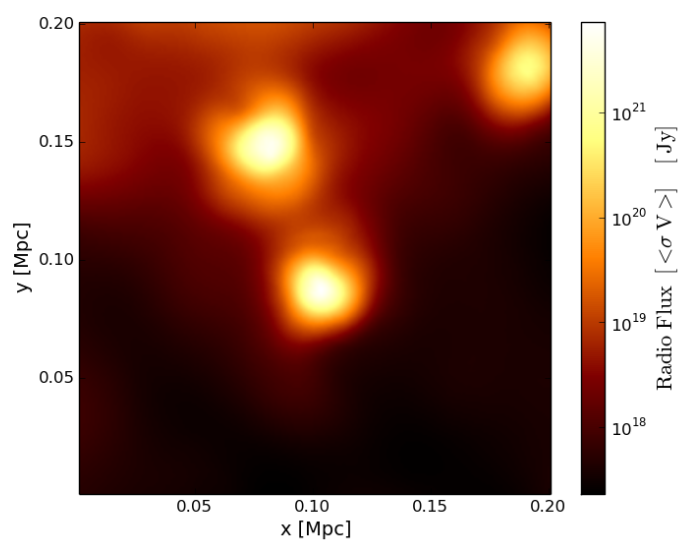

Figure 30: Synchrotron emission map of DM sub-halo 2 at $110 \mathrm{MHz}$.

\begin{tabular}{|l|c|c|c|}
\hline Halo ID & M/L & $\begin{array}{l}\text { Maximum density of } \\
\mathrm{DM}\left[\mathrm{M}_{\odot} \mathrm{kpc}^{-3}\right]\end{array}$ & $\begin{array}{l}\text { Total mass of the halo in } \\
30 \mathrm{kpc} \text { radius }\left[\mathrm{M}_{\odot}\right]\end{array}$ \\
\hline DM sub-halo 1 & 30.5 & $3.4 \times 10^{5}$ & $3.18 \times 10^{10}$ \\
\hline DM sub-halo 2 & 317 & $5.8 \times 10^{6}$ & $3.01 \times 10^{10}$ \\
\hline
\end{tabular}

Table 3: Halo ID, maximum DM density summed along the line-of-sight and total mass with in 30 $\mathrm{kpc}$ radius for the two high $\mathrm{M} / \mathrm{L} \mathrm{DM}$ sub-halos considered in our study.

\begin{tabular}{|l|l|l|l|l|}
\hline Halo ID & $\begin{array}{c}\text { Gas } \\
\text { fraction }\end{array}$ & $\begin{array}{c}\text { Star } \\
\text { fraction }\end{array}$ & $\begin{array}{c}\text { DM } \\
\text { fraction }\end{array}$ & $\begin{array}{c}\text { Radial } \\
\text { distance }[\mathrm{Mpc}]\end{array}$ \\
\hline DM sub-halo 1 & 0.00594977 & 0.03272370 & 0.96132700 & 0.9785210 \\
\hline DM sub-halo 2 & 0.00944456 & 0.00314819 & 0.98740700 & 1.2760991 \\
\hline
\end{tabular}

Table 4: Halo ID, fraction of gas, star and DM in the halo with in $30 \mathrm{kpc}$ radius, and radial distance from the center of the cluster for the two high M/L DM sub-halos considered in our study.

Figure 31 shows the radio flux density of DM sub-halo 1 and 2 in red and cyan color, respectively. The flux densities for both DM sub-halos are above the SKA sensitivity shown in magenta color [11]. In Fig. 32 we give the scaled comparison of the flux densities of DM sub-halo 1 and 2 with the observation of the Coma cluster (blue data points) from [12], they both fit in most part of the data with a little deviation at higher frequencies. 


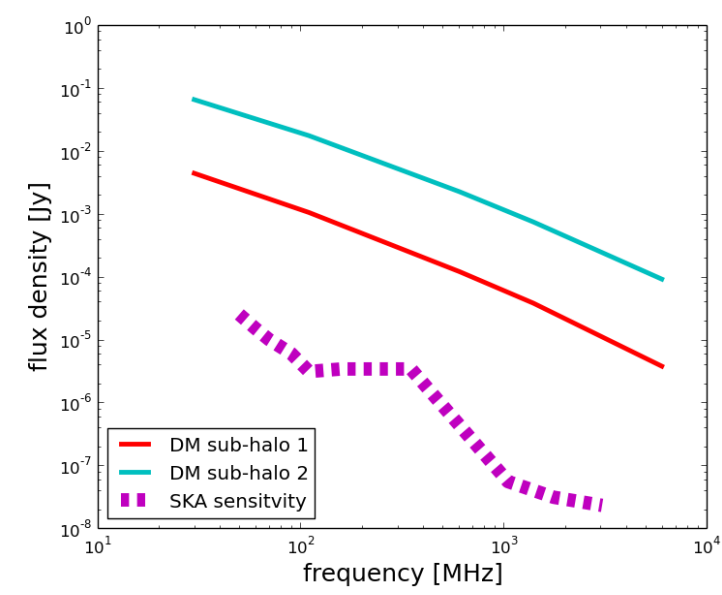

Figure 31: The radio flux density of the two DM sub-halos in comparison with the SKA sensitivity from [11].

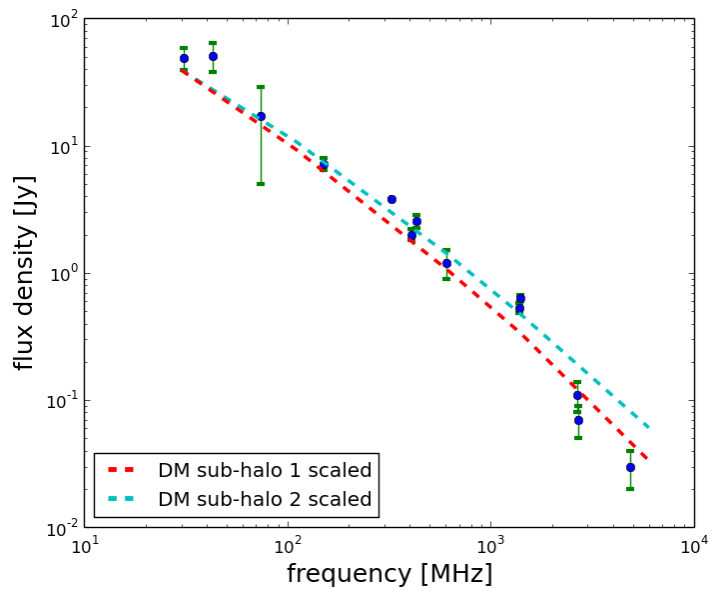

Figure 32: Scaled comparison of spectral feature of the two DM sub-halos with observational data of the Coma cluster (blue data points).

\section{High M/L sub-halos and the gamma-ray emission from DM annihilation processes.}

In the following we discuss some of the results we have obtained for the gamma-ray emission maps and flux densities for the three high M/L DM sub-halos we have chosen, whose properties are listed in table 3 and 4. These calculation are made for neutralino mass of $\mathrm{M}_{\chi}, 35 \mathrm{GeV}$ and velocity averaged DM annihilation cross-section, $\langle\sigma V\rangle$, of $1.0 \times 10^{-26} \mathrm{~cm}^{3} \mathrm{~s}^{-1}$ from Fermi upper limit.

The emission expected from DM annihilation process forming neutral pions and consequentially decaying to gamma-rays is often said to be the cleanest signal from the DM annihilation process, as they are not affected by the interaction of the halo environment such as the magnetic fields of the intra-cluster medium. The gamma-ray emission maps from the pion decay process at $100 \mathrm{MeV}$ for DM sub-halo 1 and 2 are given in Figs. 33, 34. These maps look exactly like the corresponding maps of the square of the DM density (Figs. 25 and 27), which justify the fact that the gamma-rays from the pion decay process are the cleanest signal expected from the DM annihilation process. In Figs. 35 and 36 we show the gamma-ray emissions from the non-thermal bremsstrahlung process at $100 \mathrm{MeV}$ for DM sub-halo 1 and 2. Comparing between the emission maps of the gamma-rays produced by the two emission processes we find $\sim 6$ and 5 orders of magnitude drop for DM sub-halo 1 and 2 as the values in the color bar indicate, respectively. We interpret these as the effect of the gas content which is required in the determination of the nonthermal bremsstrahlung process, which have resulted in the lower flux values for the gamma-rays from the non-thermal bremsstrahlung process.

In Fig. 37 we show the gamma-ray differential flux from pion decay and non-thermal bremsstrahlung process for the two high M/L DM sub-halos whose DM and gas density square maps integrated along the line-of-sight is given from Fig. 25 to 28. The pion decay shown by the sold curves in Fig. 37 stays above the bremsstrahlung flux values shown in dashed curves for almost all energies between the mass of electron, $\mathrm{m}_{e}, 511 \mathrm{keV}$ and the mass of neutralino $\mathrm{M}_{\chi}, 35 \mathrm{GeV}$ for both $\mathrm{DM}$ 


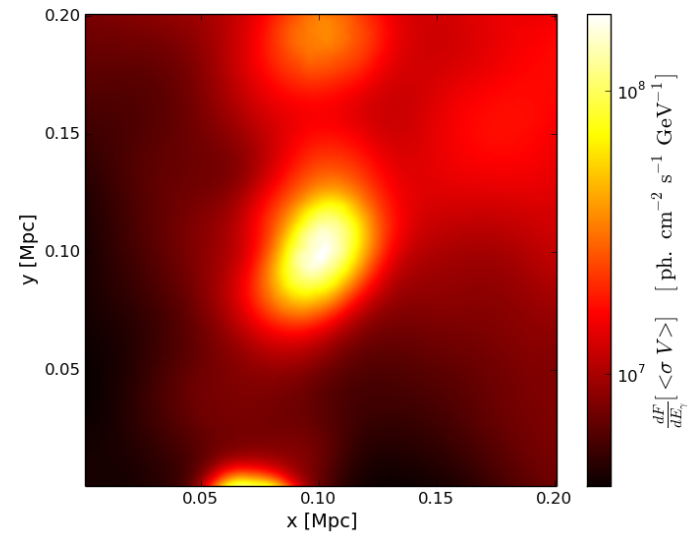

Figure 33: Gamma-ray emission map from pion decay at $100 \mathrm{MeV}$ for DM sub-halo 1.

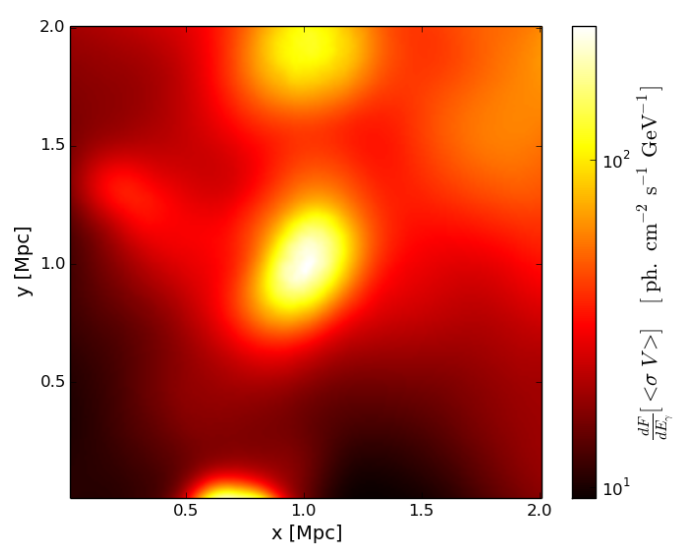

Figure 35: Gamma-ray emission map from non-thermal bremsstrahlung process at $100 \mathrm{MeV}$ for DM sub-halo 1.



Figure 34: Gamma-ray emission map from pion decay at $100 \mathrm{MeV}$ for DM sub-halo 2 .

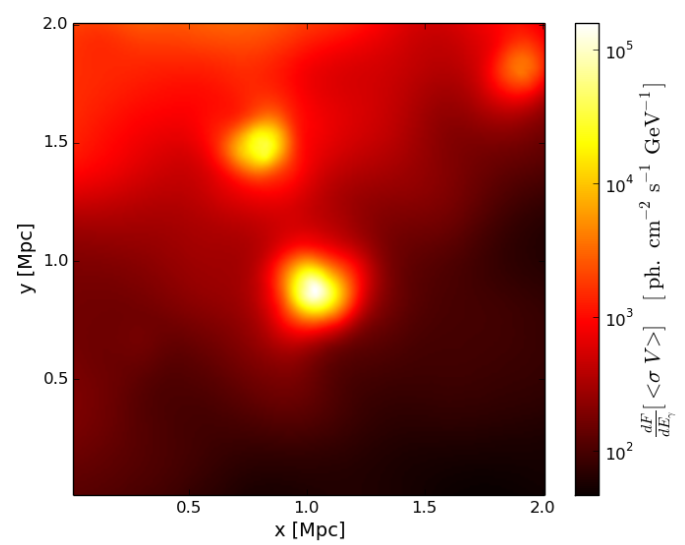

Figure 36: Gamma-ray emission map from non-thermal bremsstrahlung process at $100 \mathrm{MeV}$ for DM sub-halo 2.

sub-halos. This is because the gamma-ray flux from pion decay is the initial gamma-ray spectrum multiplied by the square of the DM density values integrated along the line-of-sight. While these DM sub-halos have high DM densities or DM fractions as shown in Tabs. 3 and 4 they also show much lower fraction of gas content which enters in to the calculation of thermal electron density. The later is also required to calculate the non-thermal bremsstrahlung emissions, hence it produces a lower value of non-thermal bremsstrahlung flux as compared to the gamma-ray flux from pion decay. As a result the gamma-ray flux from high $\mathrm{M} / \mathrm{L}$ sub-halos will be dominantly contributed from pion decay processes as shown in Fig. 37 with a combined gamma-ray flux density from IC scattering (even though not shown here), non-thermal bremsstrahlung and pion decay processes having a close resemblance to the flux densities from pion decay process. 


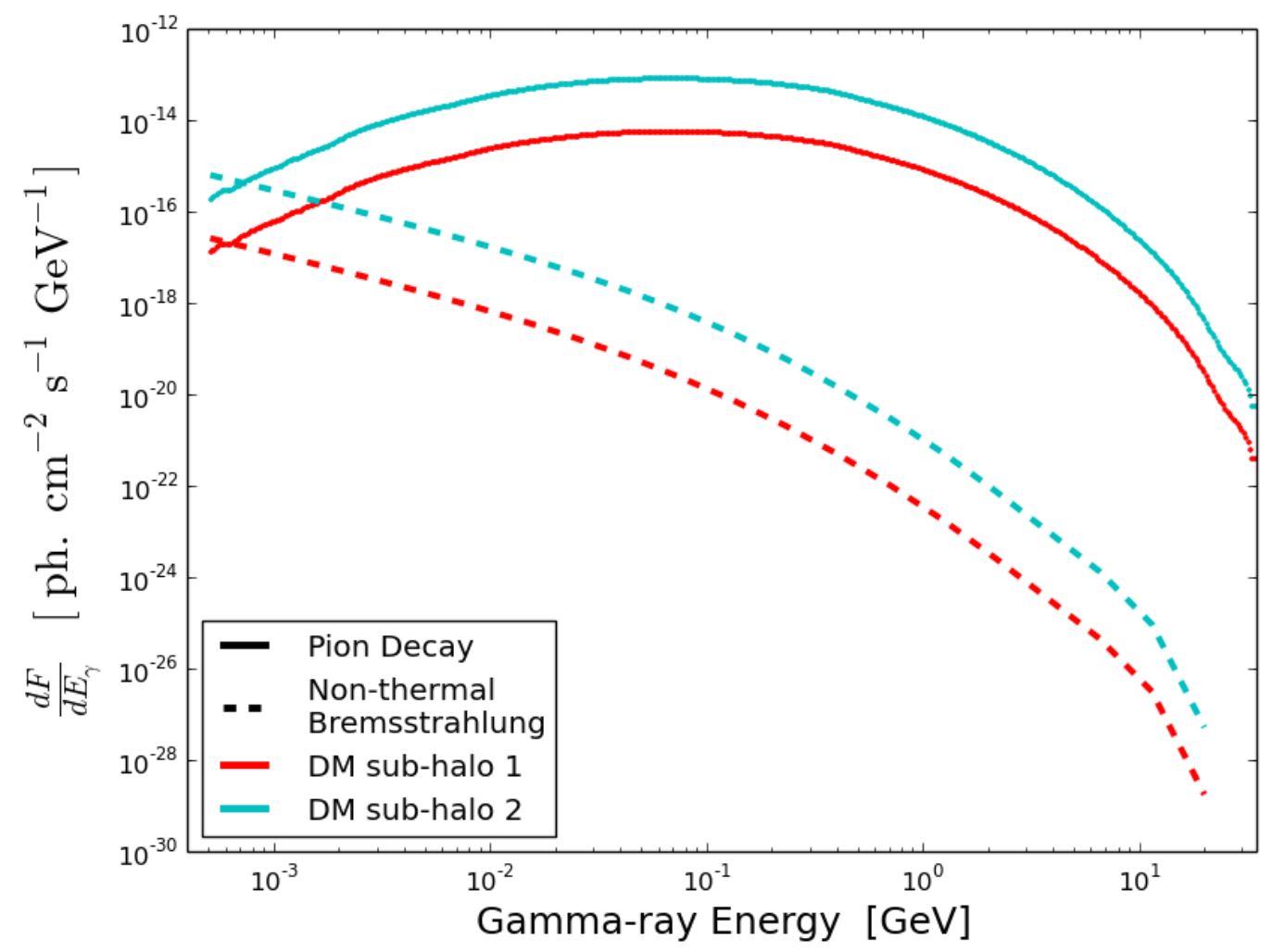

Figure 37: Differential gamma-ray flux of DM sub-halos 1 and 2 from pion decay and non-thermal bremsstrahlung processes.

\section{Conclusions and future work}

Most of the matter in the universe is in the form of non-luminous DM. The particle nature of DM is still unknown. DM self-annihilation would provide a mechanism for indirect detection via electromagnetic emission from secondary electrons production. The spectral features of the emission are expected to be closely related to the nature, composition and mass of the DM particles [4]. We determine the radio and gamma-ray emissions from neutralino DM annihilation processes considering two neutralino masses, $\mathrm{M}_{\chi}=35 \mathrm{GeV}$ and $60 \mathrm{GeV}$ along with two different models of magnetic fields.

Using the Marenostrum-MultiDark SImulation of galaxy Clusters (MUSIC-2) [5], we develop a semi-analytical model which provide multi-wavelength emission maps from DM annihilation processes in galaxy clusters and sub-halos. The high resolution and number of MUSIC-2 clusters allows us to single out DM sub-halos where it can be expected that the DM annihilation signal exceeds ordinary emission. Applying a Smooth Particle Hydrodynamics (SPH) kernel allows us to determine the DM density at arbitrary locations within the cluster volume which is used to focus on the contribution of sub-halos to the multi-wavelength emission maps of clusters.

The DM radio flux densities for a neutralino mass of $35 \mathrm{GeV}$ and the magnetic field based 
on Model A match best the observed diffuse radio emission of Coma [12]. The fact that the flux density shows a very good agreement with the observed one without invoking a boost factor from DM sub-structures shows that the distribution of sub-structures is described well in the simulations which boosted the signals from DM annihilation processes exactly to the level of observed flux. Our model also predicts gamma-ray emission from pion decay, non-thermal bremsstrahlung and IC scattering processes. The contribution to the overall gamma-ray flux from IC dominates at low energies. Non-thermal bremsstrahlung is most prominent at intermediate energies. At energies above $\sim 35 \mathrm{MeV}$ pion decay dominates.

In order to investigate the annihilation signal from "naked" sub-halos we select sub-halos with exceptionally high mass to light ratios from all 282 MUSIC-2 clusters. These sub-halos are expected to be good candidates for studying the nature of DM via the indirect detection of signals from secondary electrons. The radial distribution of high M/L DM sub-halos is more strongly peaked at $\sim \mathrm{R}_{200 \text { crit }}$ compared to the overall distribution of sub-halos.

We demonstrate that the non-thermal emission of DM annihilation from three selected subhalos is above the sensitivity limit of the Square Kilometer Array (SKA) with an integration time of 1000 hours. The gamma-ray flux densities of high M/L sub-halos show relatively low gamma-ray flux from non-thermal bremsstrahlung. In some cases their non-thermal bremsstrahlung spectrum stays below the pion decay spectrum at almost all energies. Consequently, the overall gamma-ray flux spectrum from high $\mathrm{M} / \mathrm{L}$ sub-halos is dominated by the pion decay process.

In the current work we have considered the energy loss of secondary electrons as they interact with the ICM. However, we have not taken into account the diffusion of secondary electrons which would be required for a more realistic study of DM annihilation signal from sub-halos. Also, small amounts of baryons in the high $\mathrm{M} / \mathrm{L}$ sub-halos may contaminate the annihilation signal which needs to be determined quantitatively. Finally, comparison of our results with observations in the gamma-ray band is required to confirm our DM annihilation signal predictions. Very recently, a gamma-ray detection from a DM sub-halo has been reported by [18]. The $\gamma$-ray spectrum they present is consistent with DM annihilation into final state $b \bar{b}$ also used in our model. This opens the opportunity for a future comparison with our model prediction.

\section{Acknowledgments}

This work is supported by the South African Research Chairs Initiative of the Department of Science and Technology and National Research Foundation of South Africa. R. R. Mekuria acknowledges the financial support he received from the DST/NRF SKA-research chair grant of S. Colafrancesco. P. M. acknowledges support from the DST/NRF SKA post-graduate bursary initiative. 


\section{Appendix A}



Figure 38: Radio emission map at $110 \mathrm{MHz}$ using magnetic field of Model A for DM mode $35 \mathrm{GeV}$.

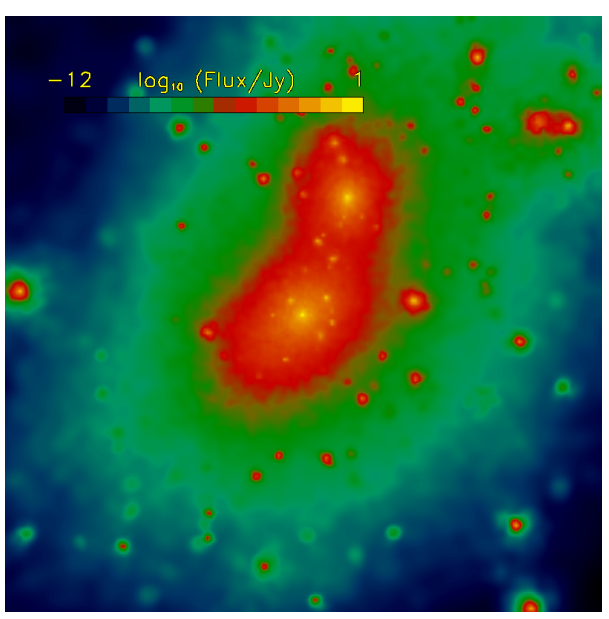

Figure 40: Radio emission map at $110 \mathrm{MHz}$ using magnetic field of Model B for DM model $35 \mathrm{GeV}$.

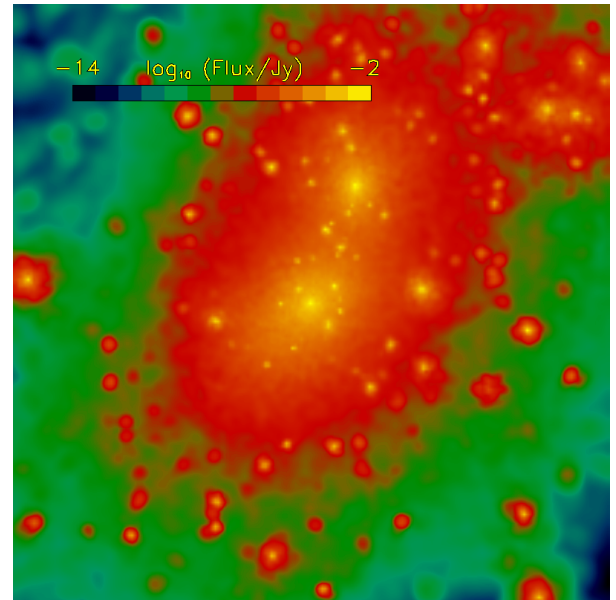

Figure 39: Radio emission map at $1400 \mathrm{MHz}$ using magnetic field of Model A for DM model $35 \mathrm{GeV}$.

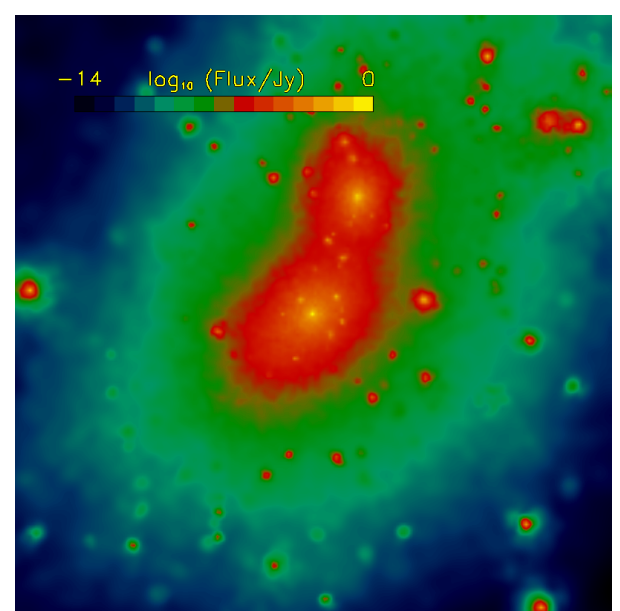

Figure 41: Radio emission map at $1400 \mathrm{MHz}$ using magnetic field of Model B for DM model $35 \mathrm{GeV}$. 


\section{References}

[1] Vikhlinin, A., Kravtsov, A., Forman, W., et al. 2006, Astrophysical Journal, 640, 691

[2] Ryu, D., Kang, H., Hallman, E., \& Jones, T. W. 2003, Astrophysical Journal, 593, 599

[3] Kolb, E. W., \& Turner, M. S. 1990, Front. Phys., Vol. 69

[4] Colafrancesco, S., Profumo, S., \& Ullio, P. 2006, Astronomy and Astrophysics, 455, 21

[5] Sembolini, F., Yepes, G., De Petris, M., et al. 2013, Monthly Notices of the RAS, 429, 323

[6] Springel, V. 2010, Annual Review of Astron and Astrophys, 48, 391

[7] Colafrancesco, S ., Marchegiani, P., \& Beck, G. 2015, Journal of Cosmology and Astroparticle Physics, 2, 032

[8] Bonafede, A., Feretti, L., Murgia, M., et al. 2010, Astronomy and Astrophysics, 513, A30

[9] Longair, M. S. 1994, High energy astrophysics. Volume 2. Stars, the Galaxy and the interstellar medium., by Longair, M. S.. Cambridge University Press, Cambridge (UK), 1994, 410 p.

[10] Colafrancesco, S., Lieu, R., Marchegiani, P., et al. 2011, Astronomy and Astrophysics, 527, A80

[11] Dewdney, P., Turner, W., Millenaar, R., McCool, R., Lazio, J. \& Cornwell, T., 2012, SKA baseline design document, http: / / www. skatelescope.org/wp-content/uploads/2012/07/ SKA-TEL-SKO-DD-001-1_BaselineDesign1.pdf

[12] Thierbach, M., Klein, U., \& Wielebinski, R. 2003, Astronomy and Astrophysics, 397, 53

[13] Marchegiani, P., \& Colafrancesco, S. 2016, arXiv:1610.00568

[14] Hooper, D., Linden, T., \& Mertsch, P. 2015, Journal of Cosmology and Astroparticle Physics, 3, 021

[15] Ackermann, M., Ajello, M., Albert, A., et al. 2016, Astrophysical Journal, 819, 149

[16] Markevitch, M., Gonzalez, A. H., Clowe, D., et al. 2004, Astrophysical Journal, 606, 819

[17] Behroozi, P. S., Wechsler, R. H., \& Wu, H.-Y. 2013, Astrophysical Journal, 762, 109

[18] Wang, Y.-P., Duan, K.-K., Ma, P.-X., et al. 2016, arXiv:1611.05135 